\title{
Photorepair of RNA polymerase arrest and apoptosis after ultraviolet irradiation in normal and XPB deficient rodent cells
}

\author{
V Chiganças ${ }^{1}$, LFZ Batista ${ }^{1}, \mathrm{G}$ Brumatti ${ }^{2}$, \\ GP Amarante-Mendes ${ }^{2}$, A Yasui ${ }^{3}$ and CFM Menck ${ }^{*}, 1$ \\ 1 Departamento de Microbiologia, Instituto de Ciências Biomédicas, \\ Universidade de São Paulo 05508-900, SP, Brazil \\ 2 Departamento de Imunologia, Instituto de Ciências Biomédicas, Universidade \\ de São Paulo and Instituto de Investigação em Imunologia, Instituto do Milênio \\ 05508-900, SP, Brazil \\ ${ }^{3}$ Department of Molecular Genetics, Institute of Development, Aging and \\ Cancer, Tohoku University, Sendai 980-8575, Japan \\ * Corresponding author: Dr. CFM Menck, Depto. de Microbiologia, ICB, USP, \\ Av. Prof. Lineu Prestes, 1374. Ed. Biomédicas 2, São Paulo, 05508-900, SP. \\ Tel. 55.11.3091.7499; Fax 55.11.3091.7354; E-mail: cfmmenck@usp.br
}

Received 4.1.02; revised 1.3.02; accepted 3.5.02

Edited by G Nuñez

\begin{abstract}
Cyclobutane pyrimidine dimers (CPDs) are directly involved in signaling for UV-induced apoptosis in mammalian cells. Failure to remove these lesions, specially those located at actively expressing genes, is critical, as cells defective in transcription coupled repair have increased apoptotic levels. Thus, the blockage of RNA synthesis by lesions is an important candidate event triggering off active cell death. In this work, wild-type and XPB mutated Chinese hamster ovary (CHO) cells expressing a marsupial photolyase, that removes specifically CPDs from the damaged DNA, were generated, in order to investigate the importance of this lesion in both RNA transcription blockage and apoptotic induction. Photorepair strongly recovers RNA synthesis in wild-type $\mathrm{CHO}$ cell line, although the resumption of transcription is decreased in XPB deficient cells. This recovery is accompanied by the prevention of cells entering into apoptosis. These results demonstrate that marsupial photolyase has access to CPDs blocking RNA synthesis in vivo, and this may be affected by the presence of a mutated XPB protein.

Cell Death and Differentiation (2002) 9, 1099-1107. doi:10.1038/sj.cdd.4401072
\end{abstract}

Keywords: Cyclobutane pyrimidine dimers; photoreactivation; apoptosis; RNA transcription

Abbreviations: (6-4) PP, (6-4) photoproduct; CPD, cyclobutane pyrimidine dimer; $\mathrm{CHO}$, Chinese hamster ovary; $\mathrm{CS}$, Cockayne's syndrome; ESS, endonuclease sensitive sites; GGR, global genomic repair; NER, nucleotide excision repair; PI, propidium iodide; PRL, photoreactivating light; TCR, transcription-coupled nucleotide excision repair; TFIIH, transcription factor $\mathrm{H}$ of RNA polymerase II; TTD, trichothiodystrophy; XP, xeroderma pigmentosum

\section{Introduction}

The main photoproducts generated by UV irradiation (UV-C light, $254 \mathrm{~nm}$ ), cyclobutane pyrimidine (CPDs) and pyrimidine (6-4) pyrimidone dimers (6-4 PPs), can induce an active cell death process known as apoptosis in mammalian cells. ${ }^{1,2}$ These DNA lesions are normally repaired by nucleotide excision repair (NER), involving two sub pathways, one that discriminates lesions present in the transcribed strand of active genes (transcription-coupled nucleotide excision repair, TCR), and the other that removes lesions from the rest of the genome (global genomic repair, GGR) ${ }^{3,4}$ There are several human syndromes related to defective NER proteins, which affect individual cancer proneness and even organism development. Xeroderma pigmentosum (XP) patients, for example, display high sunlight sensitivity and dramatically increased incidence of skin cancer, often linked with neurological abnormalities. Cells from most of these patients have been described as deficient in their DNA repair (NER) abilities and seven complementation groups were identified, corresponding to seven different genes (XP genes). The Cockayne's syndrome (CS) and trichothiodystrophy (TTD) are further examples of NER deficiencies, whose clinical features are also sun sensitivity and mental retardation. ${ }^{5}$ Patients with CS have a phenotype related to impaired transcriptional activity and, in contrast to XP, generally do not develop cancer, presumably because of the elimination of cells with DNA damage by apoptosis. ${ }^{6}$ Mutations in the CSA and CSB genes were identified in most of the CS patients, and CS cells have been shown to be deficient in RNA synthesis recovery after UV and, more specifically, on the removal of CPDs by TCR. ${ }^{4}$

One of the important responses of UV irradiated mammalian cells is cell killing by the induction of an active process of cell death, also known as apoptosis. ${ }^{7}$ DNA damages, mainly CPDs, were shown to be a necessary signal of UV-induced apoptosis in human cells, as their removal by photorepair prevents cell death. ${ }^{2}$ Besides being a target for DNA repair, these lesions obstruct DNA replication and RNA synthesis, which can be upstream in signaling the cascade that leads to apoptosis. DNA synthesis inhibition by aphidicolin in UV irradiated rat kangaroo cells did not affect cell death responses, indicating that signals to apoptosis occur independently of DNA replication of damaged template. ${ }^{8}$ In contrast, studies with hamster cells suggest that unrepaired DNA lesions trigger apoptosis, by their conversion to double-strand breaks in the cell cycle progression after UV-C irradiation. ${ }^{9}$ On the other hand, human cells lacking TCR undergo apoptosis at lower UV doses than cells proficient in strandspecific repair. The increased apoptosis is paralleled by increased inhibition of RNA synthesis and accumulation of 
the tumor suppressor p53 protein. ${ }^{6}$ This protein has also been shown to interact with XPB and XPD proteins, two components of the transcription factor $\mathrm{H}$ of RNA polymerase II (TFIIH complex), which participate directly in RNA transcription and NER and, as a result, may be involved in the apoptotic pathway. ${ }^{10}$ These results lead to the proposition of the blockage of RNA polymerase activity at DNA lesions. In fact, CPDs represent physical hindrance to RNA polymerase II progression. ${ }^{11}$ The interruption of the transcriptional complex by these photoproducts may be the signal for UV-induced apoptosis, indicating that persistent lesions in the transcribed strand promote cellular suicide. ${ }^{12}$ More recently, it was found that the recovery of RNA synthesis might precede the removal of photolesions in the transcribed strand ${ }^{13}$ or even that this recovery is independent of TCR. ${ }^{14}$

In the present work, the role of CPD lesions in the early signals for UV induced programmed cell death was investigated. This was achieved by employing wild-type and DNA repair deficient (XPB mutated) Chinese hamster ovary $(\mathrm{CHO})$ cell lines expressing high levels of the heterologous CPD photolyase gene (from Potorous tridactylus), which were confirmed to be proficient in photorepair. These cells were used to directly explore the effects of the removal of CPDs on apoptosis and RNA synthesis inhibition. The results indicate that marsupial photolyase has free access to CPDs in wild-type rodent cells even when the lesion is obstructing the RNA polymerase complex, significantly reducing UV harmful effects. The data support the hypothesis that the RNA polymerase complex may act as sensor of CPDs, and that this process is an important and necessary intermediate for triggering cellular suicide. As expected, cells deficient in DNA repair (XPB mutation) suffer apoptosis at lower UV doses. However, the removal of CPDs by photolyase was less efficient in these mutated cells with a decreased recovery in RNA synthesis inhibition. It is possible that the mutated $X P B$ protein may shield the damage at the stalled transcription complex, limiting the access of the photolyase.

\section{Results}

\section{Selection of $\mathrm{CHO}$ cells proficient in photorepair}

Wild-type and $X P B$ mutated $\mathrm{CHO}$ cells were transfected with the marsupial photolyase gene (phr) and clones tested for their ability to perform photorepair. Individual clones that showed a better survival recovery when exposed to photoreactivating light (PRL) after UV irradiation are shown in Figure 1A and were used throughout this work. Cell death occurred in a dose-dependent manner in both cell types, but $\mathrm{CHO}-X P B$ cells exhibited a higher sensitivity to UV, a phenotypic response corresponding to deficiency in DNA repair. After photoreactivation, the clones transfected with the phr gene exhibited an increased survival response in comparison to cells maintained in the dark or to parental cells. The differences in cell survival become more evident at higher UV doses, as observed at $15 \mathrm{~J} / \mathrm{m}^{2}$ for wild-type and at $5 \mathrm{~J} / \mathrm{m}^{2}$ for $X P B$ mutated cells. This survival recovery after PRL was probably due to the removal of CPDs by the marsupial CPD photolyase, confirming the heterologous action of this protein in rodent cells.

The expression of the gene phr in $\mathrm{CHOphr}$ and $\mathrm{CHO}-$ $X P B p h r$ cell lines were investigated by Northern blot experiments (Figure 1B). Similar very high levels of the marsupial photolyase mRNA were detected in both cell lines. The housekeeping gene GAPDH was used as a control probe for comparison.

\section{Removal of CPDs by photorepair}

The number of CPDs after UV-irradiation was determined as the number of T4-endonuclease sensitive sites (ESS). This enzyme recognizes and nicks the DNA at the CPD site, and the reduction of the DNA molecular weight is detected by sedimentation in alkaline sucrose gradients. Thus, the frequency of ESS corresponds to the relative frequency of CPDs in the cellular DNA. ${ }^{15}$ These values were used to calculate the efficiency of CPD removal by photorepair. The data is presented in Table 1. As expected, there is a significant reduction in the number of ESS after PRL in cells carrying the phr gene. However, there is a clear difference in the ability of photorepair of wild-type and XPB mutated cells lines. While in wild-type cells the removal of CPDs is almost complete after photoreactivation, in $\mathrm{CHO}-X P B p h r$ cells efficiency is reduced to approximately $60 \%$.

\section{Prevention of UV-induced apoptosis by photorepair}

Apoptotic cells exhibit a typical oligonucleosomal DNA degradation pattern that, together with the condensation of chromatin, results in the appearance of subdiploid nuclei. This apoptotic endpoint was quantified by flow cytometry analysis of the DNA content of individual nuclei $48 \mathrm{~h}$ after UV irradiation (Figures 2 and 3). Dose-dependent cell death can be observed in both $\mathrm{CHO}$ and $\mathrm{CHO}-X P B$ cells. Cells defective in the $X P B$ gene (Figure 3 ) exhibit a higher percentage of programmed cell death at lower UV doses, when compared to wild-type cells (Figure 2). These results confirm that NER deficiency makes these cells more sensitive to UV induced-apoptosis. When photorepair was performed in cells expressing the marsupial photolyase in PRL conditions, apoptosis was strongly decreased in comparison to parental cells or samples maintained in the dark. It is important to notice that in cells that do not carry the phr gene there is no significant difference in the frequency of apoptotic cells, whether the cells are submitted or not to fluorescent light. These data indicate that heterologous photorepair of CPDs prevents UVinduced apoptosis in the cell lines $\mathrm{CHOphr}$ and $\mathrm{CHO}-$ $X P B p h r$. In order to invetigate if photoreactivation is specific for UV-irradiation, apoptosis was induced by treating the cells with staurosporine $100 \mu \mathrm{M}$ (serine/ threonine kinase inhibitor). ${ }^{16}$ As expected, PRL did not result in the prevention of apoptosis in any of the cell lines (data not shown). These observations confirm that the protection of photorepair is specific for this UV-induced apoptosis. 

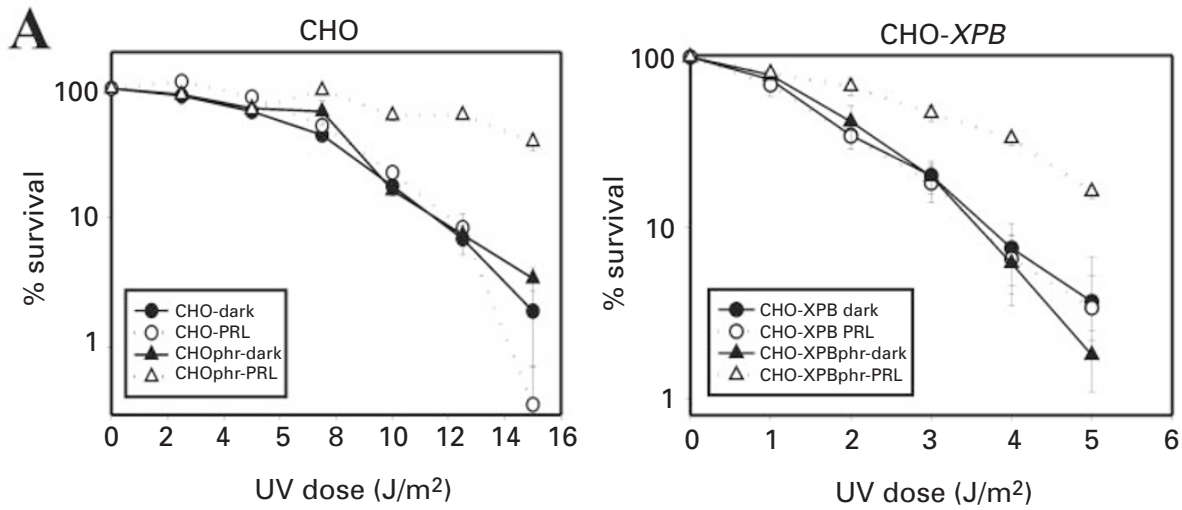

B

Figure 1 Photolyase effects in cell survival and mRNA expression in $\mathrm{CHO}$ transfected cells. (A) Low density cultures of cells not transfected (solid lines) or expressing the phr gene (dotted lines) were UV irradiated with the indicated doses and then maintained in the dark (closed symbols) or under fluorescent light illumination (PRL; open symbols) in prewarmed PBS during $2 \mathrm{~h}$. The left panel corresponds to DNA repair proficient cells (CHO) and the right panel to XPB deficient cells $(\mathrm{CHO}-X P B)$, as indicated. The data represent the average of two independent experiments performed in triplicate. The bars indicate standard deviation and the differences between experimental (cells expressing phr and under PRL) and control samples were assessed for statistical significance by Student's $t$-test $(P<0.05)$. (B) Expression of marsupial photolyase mRNA by Northern blot. Right panel: samples of $5 \mu \mathrm{g}$ total RNA from the different cell lines were resolved by denaturing agarose gel electrophoresis, hybridized with specific probes (photolyase gene, upper panel, or GAPDH gene, lower panel), and then analyzed by autoradiography. Cell lines expressing the marsupial phr gene display a $1.8 \mathrm{~Kb}$ RNA. The housekeeping gene GAPDH (0.49 Kb) was used as an internal control. Cell lines are indicated. The left panel is a positive control of hybridization, with $50.0,5.0$ and 0.5 ng of the probe (photolyase DNA) applied in the gel

\section{Inhibition of RNA synthesis is rescued by photorepair}

Several reports have shown that transcriptional synthesis is decreased after UV irradiation, this effect being more prominent in cells with TCR deficiency, such XPB deficient cells. ${ }^{6,12}$ Detection of ${ }^{3} \mathrm{H}$-rNTPs incorporation in $\mathrm{CHO}$ cells was used as an indicator of transcriptional synthesis. The results are shown in Figure 4. $\mathrm{CHO}$ and $\mathrm{CHOphr}$ cells exhibit a strong inhibition of RNA synthesis by irradiation of UV light. When CHOphr cells were exposed to PRL conditions, they displayed a significant recovery of RNA synthesis in comparison with dark conditions and to cells not bearing the photolyase gene (Figure 4A). This recovery was decreased when PRL was not performed immediately after UV. Nevertheless, even $4 \mathrm{~h}$ after irradiation, the cells exposed to PRL still showed a $50 \%$ recovery of the RNA incorporation. For $\mathrm{CHO}-X P B p h r$ cells, there was also a strong inhibition of RNA transcription at lower doses of UV irradiation (Figure 4B), confirming previous observations that RNA synthesis is directly affected in cells lacking TCR. However, the recovery of RNA transcription by photorepair was much less efficient for $\mathrm{CHO}-X P B$ cells than that observed for DNA repair proficient $\mathrm{CHO}$ cells. It is important to point out that, in these experiments, the doses employed $\left(10.0 \mathrm{~J} / \mathrm{m}^{2}\right.$ for $\mathrm{CHO}$, and $6.0 \mathrm{~J} / \mathrm{m}^{2}$ for $\mathrm{CHO}-X P B$ cells) were chosen as they promote similar levels of RNA inhibition synthesis. As for cells proficient in DNA repair, this RNA synthesis recovery is significantly decreased when PRL was not performed immediately after UV irradiation.

\section{Discussion}

Photoreactivation is a light dependent efficient and specific type of repair, which can remove CPD or 6-4 PP from UVirradiated damaged DNA. Although no photolyase is found in placental mammals, previous reports have confirmed that heterologous expression of marsupial CPD photolyase has been found to be active in human cells, either reducing mutagenesis $^{17,18}$ or preventing UV-induced apoptosis. ${ }^{2}$ The 
Table 1 Removal of endonuclease sensitive sites (ESS) by photorepair

\begin{tabular}{|c|c|c|c|c|}
\hline \multirow[b]{2}{*}{ UV dose $\left(\mathrm{J} / \mathrm{m}^{2}\right) /$ condition } & \multicolumn{2}{|l|}{ CHOphr } & \multicolumn{2}{|l|}{ CHO-XPBphr } \\
\hline & ESS & Photorepair & ESS & Photorepair \\
\hline $1.0 / 0 \mathrm{~h}$ & 0.089 & & 0.082 & \\
\hline 1.0/dark $1 \mathrm{~h}$ & $0.080 \pm 0.012$ & & $0.092 \pm 0.052$ & \\
\hline 1.0/PRL 1h & $-0.002 \pm 0.018$ & $102 \%$ & $0.031 \pm 0.021$ & $64 \%$ \\
\hline $2.5 / 0 \mathrm{~h}$ & 0.152 & & 0.131 & \\
\hline $2.5 /$ dark $1 \mathrm{~h}$ & $0.185+0.022$ & & $0.153+0.048$ & \\
\hline 2.5/PRL $1 \mathrm{~h}$ & $0.009 \pm 0.034$ & $95 \%$ & $0.069 \pm 0.033$ & $55 \%$ \\
\hline
\end{tabular}

The ESS numbers were determined in nuclei by sedimentation in alkaline sucrose gradients as described in Material and Methods. Cells were harvested immediately after UV irradiation $(0 \mathrm{~h})$ or submitted to dark or PRL conditions $(1 \mathrm{~h})$ before ESS determination. Except for $0 \mathrm{~h}$ (single experiment), the numbers represent the average of four independent experiments (the differences between dark and PRL conditions are statistically significant; $P<0.01$, Student's $t$-test). Values for photorepair were obtained considering the decrease of ESS in cells submitted to PRL in relation to those maintained in the dark.
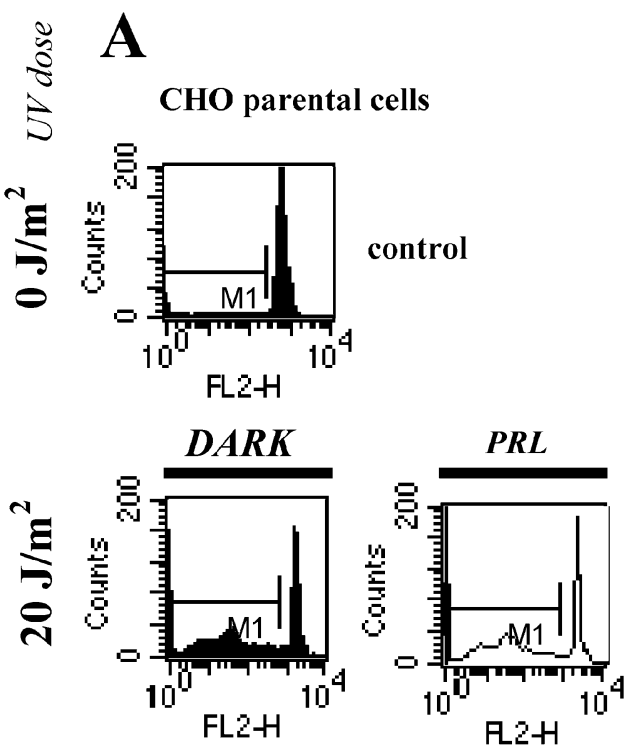

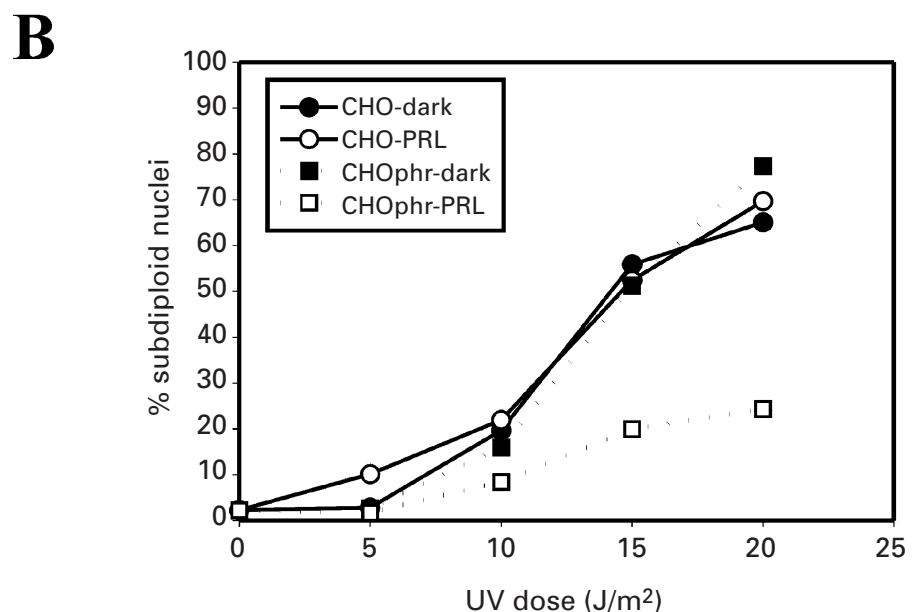

\section{CHOphr cells}
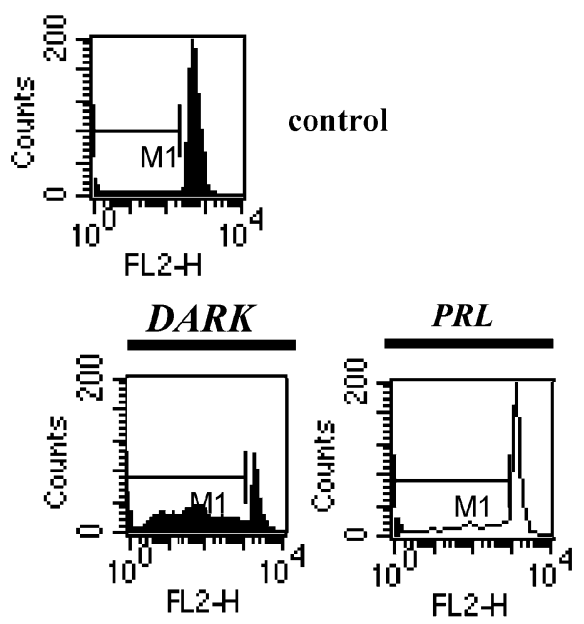

Figure 2 Photorepair prevents apoptosis in wild-type $\mathrm{CHO}$ cells. (A) Representative histograms of flow cytometry experiments for wild-type $\mathrm{CHO}$ cells. Semi confluent cells were UV irradiated at the indicated doses and maintained in the dark or PRL conditions immediately after irradiation. The subdiploid nuclei (M1) were accessed $48 \mathrm{~h}$ after UV irradiation. (B) The frequency of subdiploid nuclei was calculated and plotted for comparison. The differences between experimental and control samples were assessed for statistical significance by Student's $t$-test $(P<0.03)$

latter work indicated that CPDs are directly linked to the sequential cascade of events that lead to programmed cell death. In the present work, we intended to further investigate the early events of UV-induced apoptosis, by analyzing the role of nucleotide excision repair and the interference of CPDs in RNA transcription after UV irradiation of mammalian cells. 

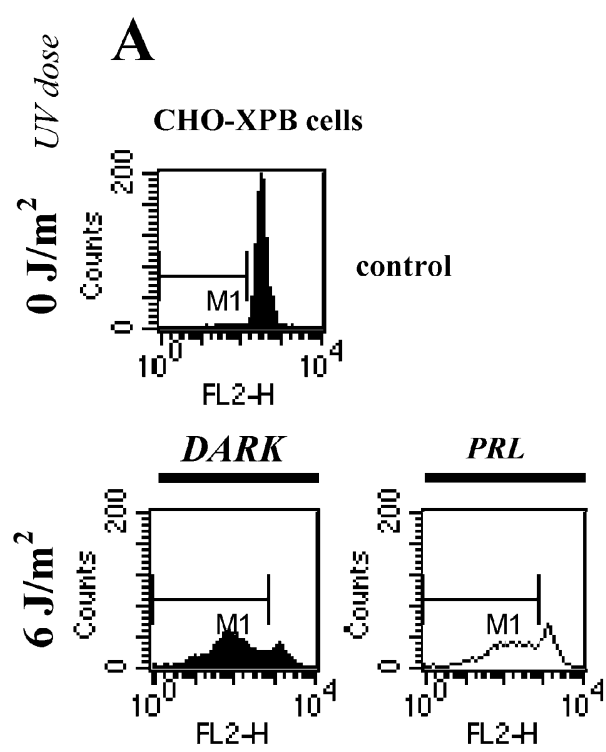

\section{CHO-XPBphr cells}
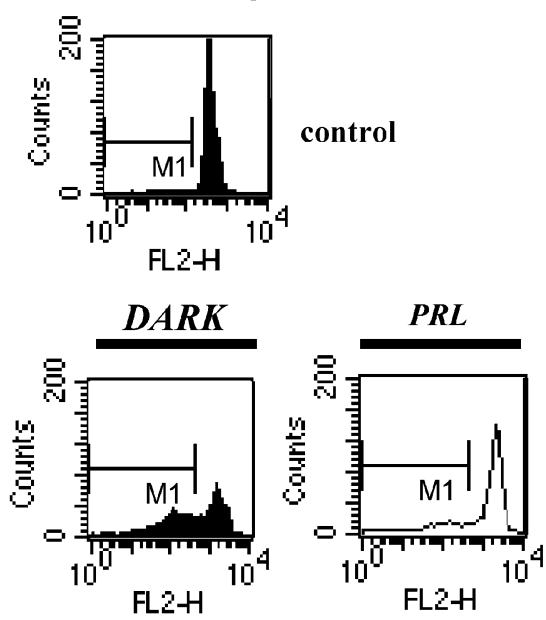

B

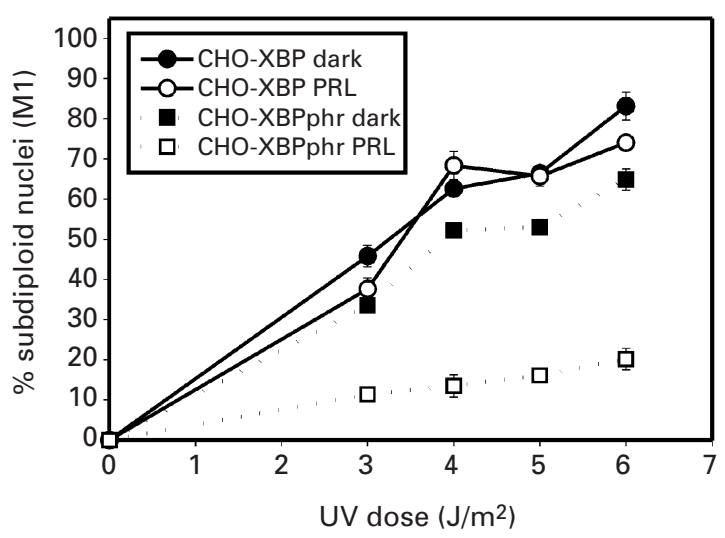

Figure 3 Photorepair prevents apoptosis in $\mathrm{CHO}-X P B$ cells. (A) Representative histograms of flow cytometry experiments with XPB mutated $\mathrm{CHO}$ cells. Sem confluent cells were UV irradiated at the indicated doses and maintained in the dark or PRL conditions immediately after irradiation. The subdiploid nuclei (M1) were accessed $48 \mathrm{~h}$ after UV irradiation. (B) The frequency of subdiploid nuclei was calculated and plotted for comparison. The differences between experimental and control samples were assessed for statistical significance by Student's $t$-test $(P<0.04)$

This was accomplished by generating $\mathrm{CHO}$ derived cell lines, including a $X P B$ deficient mutant, that express the marsupial $\mathrm{CPD}$ photolyase. The cell lines obtained, $\mathrm{CHO}$ phr and $\mathrm{CHO}-$ $X P B p h r$, proved to be informative models to study apoptosis after DNA damage and to evaluate the consequences of CPD removal to early cellular signals involved in committing the cells to death. The clones expressing the phrgene exposed to light displayed increased survival rates in comparison to those maintained in the dark (Figure 1), due to the protective action of the CPD photolyase. Similar high levels of photolyase mRNA expression were detected by Northern blot analysis in both cell lines, supporting the heterologous contribution of the enzyme in CPD removal. Using flow cytometry analysis, we observed that photorepair prevented apoptosis in phr-positive cells but not in parental cells or cells maintained in dark conditions. These data confirm that CPDs are the first signals of apoptosis in UV-irradiated cells. For $\mathrm{CHO}$ cells deficient in DNA repair (XPB cells), lower doses of UV are sufficient for the induction of apoptosis. These results are in agreement with previous reports, ${ }^{6,12}$ showing that cells deficient in TCR presented increased levels of apoptosis with low UV doses. This was interpreted as being due to increased RNA transcription arrest in TCR deficient cells, which would result in apoptotic signals. However, the data obtained for $\mathrm{CHO}-$ $X P B p h r$ cells reveal that the removal of CPDs by marsupial photolyase, although less efficient than in wild-type cells, may also prevent apoptosis even in NER-deficient cells.

It is well known that the rate of RNA transcription is decreased after DNA damage, although the molecular basis for this phenomenon and its relation to DNA repair still remains largely obscure. One possibility is that transcriptional arrest may only be a passive consequence of lesions located in the transcribed strand, blocking RNA elongation. However, the reduction in RNA synthesis may also be caused by the repression of transcription initiation ${ }^{19}$ and RNA polymerase II degradation. ${ }^{14}$ In fact, in vitro experiments indicate that the progression of RNA polymerase II in the template strand is blocked by bulky DNA 
A

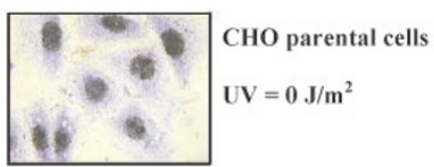

$$
\mathrm{UV}=10 \mathrm{~J} / \mathrm{m}^{2} \quad \mathrm{UV}=10 \mathrm{~J} / \mathrm{m}^{2}
$$

DARK

PRL
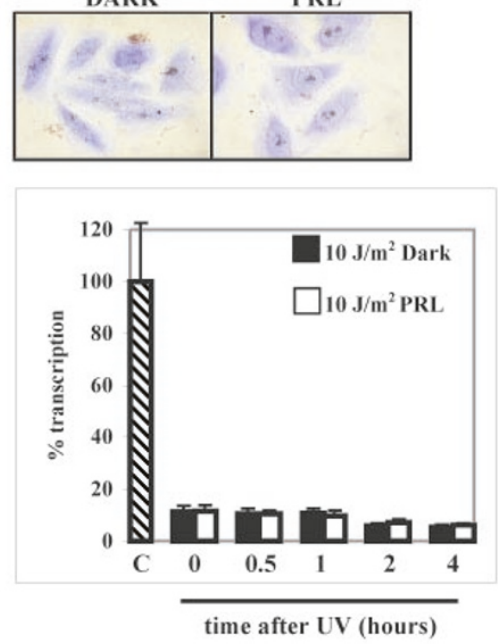

B
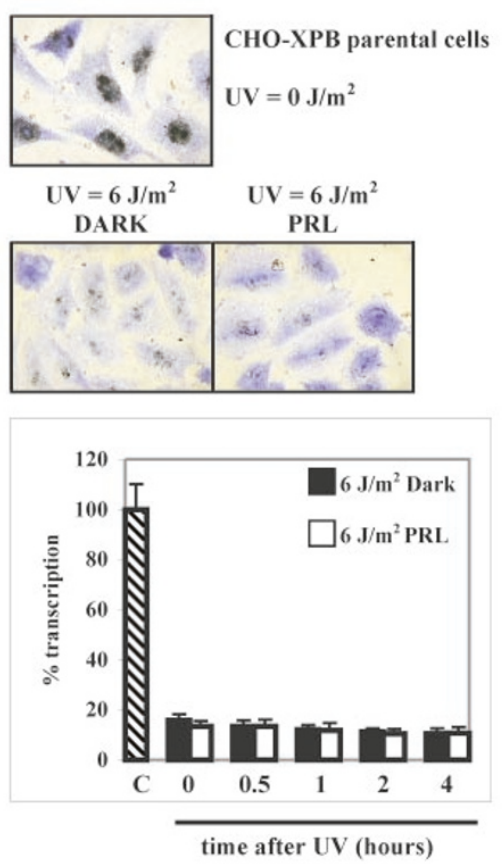
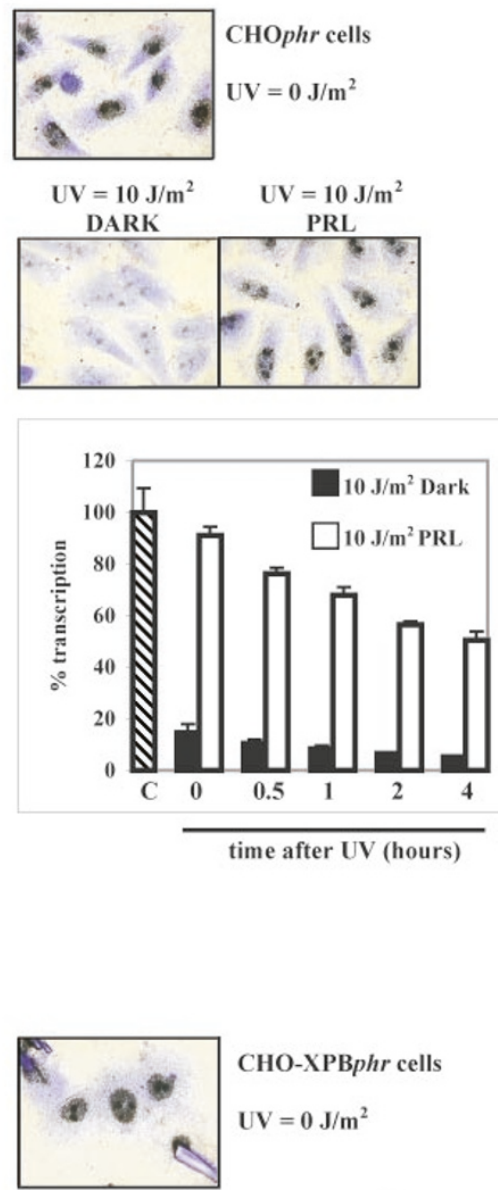

CHO-XPBphr cells

$\mathbf{U V}=\mathbf{0} \mathbf{J} / \mathbf{m}^{2}$

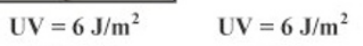

DARK

$$
\text { PRL }
$$
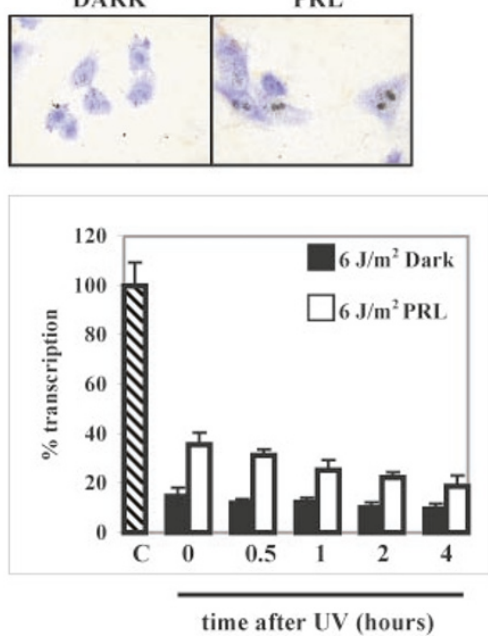

Figure 4 Photorepair recovers RNA synthesis inhibition after UV irradiation in wild-type (A) and XPB deficient (B) $\mathrm{CHO}$ cells. Approximately $1.0 \times 10^{5}$ cells were UV irradiated and then maintained in dark (closed bars) or in PRL conditions (open bars) after the indicated periods in prewarmed PBS during $1 \mathrm{~h}$. After this period, nascent RNA was pulse-labeled in a medium containing $60 \mu \mathrm{Ci} / \mathrm{ml}$ of ${ }^{3} \mathrm{H}$-uridine; the autoradiographycally induced silver grains corresponding to RNA transcription, being counted for 50 nuclei in each sample. Representative fields are shown for control samples (UV=0 J/m ${ }^{2}$, hatched bars), samples being treated immediately after irradiation. The transcriptional rate in dark or PRL conditions for all the different periods were assessed for statistical significance (Student $t$-test, $P<0.001)$

lesions such as CPDs, ${ }^{20,21}$ and the footprint of the stalled RNA polymerase II-DNA complex indicates that damage is shielded by the ternary complex covering approximately 35 base pairs around CPD. Curiously, nucleotide excision repair seems to be active even in transcription blocked dimers within the ternary complex. ${ }^{20}$ However, data 
concerning the accessibility of CPDs at transcriptionarrested sites to photolyase is more complex. Although, these lesions are normally shielded by the arrested polymerase complex, ${ }^{11}$ under certain conditions this may be displaced, allowing for photolyase access to CPD and, thus, RNA elongation in vitro. ${ }^{21}$ In this work, photoreactivation in CHOphr cells efficiently restore RNA synthesis in UV-irradiated cells. Although this restoration decreases with time after UV (e.g. $4 \mathrm{~h}$ after irradiation, photoreversion of RNA transcription is only $50 \%$ of that observed immediately after UV), these data clearly indicate that photolyase is able to remove CPDs at stalled RNA polymerases. The idea that photolyase is only removing CPDs before the transcription apparatus reaches the lesions is unlikely, as RNA synthesis inhibition reaches the maximum within the first hour after irradiation. Thus, the results presented here suggest that the photolyase has access to the CPDs at the transcriptionstalled complexes in vivo. This is in agreement with the model of RNA polymerase II displacement by accessory factors, in order to provide access to TCR enzymes. ${ }^{22-25}$

Interestingly, RNA synthesis recovery by photoreactivation is significantly decreased in $\mathrm{CHO}-X P B$ cells. It should be pointed out that these experiments were performed with doses that induce similar levels of apoptosis and RNA transcription inhibition in both cell lines (wild-type $\mathrm{CHO}$ $10.0 \mathrm{~J} / \mathrm{m}^{2}$ and $\mathrm{CHO}-X P B, 6.0 \mathrm{~J} / \mathrm{m}^{2}$ ). The rate of $\mathrm{CPD}$ removal by photorepair in the entire genome is approximately $40 \%$ lower in XPB mutated cells (Table 1 ). A simple explanation for these data could be that marsupial photolyase is less effective in CPD removal in this clone of $\mathrm{CHO}-X P B$ cells, although mRNA expression of the phr gene seems high and similar in both cell lines. A more speculative reason is, since rodent cells are GGR defective, that the chromatin context in non-transcribed regions may be less accessible to repair enzymes and, as a result, the data obtained in ESS and RRS methods would come in proximity, reflecting impairment of repair in transcribed regions in these cells. If this is correct, the reduced efficiency of photolyase in the NER deficient cells may be explained by a shielding effect of the mutated XPB protein (and probably the whole TFIIH complex) at the stalled RNA polymerase complex. Thus, the functional XPB protein may participate in the displacement of the transcription machinery, exposing the lesions, so that they can be removed by photolyase. Recently, studies in the nuclear assembly of DNA repair proteins in vivo ${ }^{26}$ demonstrated that the XPB protein is able to migrate to lesion sites even in NER deficient cells (XPA cells), but the restoration of the proteins to the pre-UV sites depends on functional NER. Thus, the differential response to recover RNA synthesis after photorepair in $\mathrm{CHO}-X P B$ cells could be a result of reduced access of lesions to DNA repair due to a shielding produced by the $X P B$ mutated protein, a possibility that remains to be determined.

Alternatively, the $X P B$ mutation may impair transcriptional resumption per se. The $X P B$ protein with its helicase function is participating in transcription initiation and in DNA repair (TCR and GGR) as a component of the TFIIH factor, $^{10,27,28}$ thereby presenting a dual function, and, consequently, $\mathrm{CHO}-X P B p h r$ cells could present a difficulty to resume transcription even after CPD removal by the heterologous photolyase. In addition to these observations, the reduced recovery of RNA synthesis in XPB mutated cells may indicate a threshold of RNA transcription inhibition signaling for UV-induced apoptosis. Once mRNA synthesis is recovered above the threshold after photorepair, the apoptotic program is prevented, as can be observed in XPB mutated cells by comparing data presented from flow cytometry analysis and RRS after $6.0 \mathrm{~J} / \mathrm{m}^{2}$ (Figures 3 and 4).

In the experiments reported here, we found a clear correlation of RNA synthesis inhibition and the induction of apoptosis. When RNA synthesis inhibition could be prevented by photorepair, there was a clear decrease in the frequency of apoptotic cells. This is in full agreement with the model that proposes RNA transcription inhibition by DNA damage as a major signal component of the apoptotic program induced by UV irradiation. ${ }^{6,12}$ This is also consistent with the increased RNA synthesis inhibition and apoptotic responses in cells mutated in the XPB gene, defective for DNA repair, specially at transcription active sites of the genome, confirming previous reports of increased apoptosis signals in TCR defective cells. ${ }^{12,29}$

In conclusion, this work is, to our knowledge, the first report of RNA synthesis recovery by photoreactivation in UV-irradiated cells, suggesting that this enzyme may have access to the CPD in the nuclear environment, even if the transcription machinery is stalled at these lesions. The RNA synthesis recovery may be a link with the decreased frequency of apoptosis in photoreactivated cells, supporting that transcription blocked by DNA lesions is a crucial event triggering downstream programmed cell death. Mutation in the $X P B$ gene, that affects DNA repair capability and transcription, impairs photoreactivation efficiency in the UV effects analyzed here by either reducing CPD exposure to photolyase or impairing transcriptional resumption even after CPD elimination.

\section{Materials and Methods}

\section{Cell Culture}

Wild-type (CHO-9) and XPB mutated (CHO-27.1) cell lines were routinely grown in DMEM (Life Technologies, Inc.) supplemented with $10 \%$ fetal bovine serum (FBS; Cultilab) and $1 \%$ antibiotic-antimycotic (Life Technologies, Inc.), at $37^{\circ} \mathrm{C}$ in a humidified $5 \% \mathrm{CO}_{2}$ atmosphere. These cell lines had been kindly provided by Dr. MZ Zolzienicka (Leiden University, The Netherlands).

\section{Generation of photolyase expressing cell lines}

$\mathrm{CHO}$ cell lines stably expressing the rat kangaroo photolyase (PHR) were generated as previously described. ${ }^{2}$ Briefly, plasmid vectors containing the marsupial phr gene and the hygromycin resistance gene were cotransfected into $\mathrm{CHO}-9$ and $\mathrm{CHO}-27.1$ cells by the standard liposomal methodology: ${ }^{30} 5 \mu \mathrm{g}$ of DNA were added to a DMEM medium with lipofectin $(1 \mathrm{mg} / \mathrm{ml}$, Invitrogen Corporation, California, USA), without serum or antibiotics, for $4 \mathrm{~h}$. Two days later, hygromycin-B (400 $\mu \mathrm{g} / \mathrm{ml}$, Invitrogen Corporation, California, USA) 
was added to the cell medium for the selection of cells that carried the exogenous gene. Individual clones were screened for those with increased UV survival after photoreactivation. Those with the best survival recovery were used in all the reported experiments.

\section{UV irradiation and clonogenic assay}

Approximately 700 cells were plated in $60-\mathrm{mm}$ Petri dishes $14-16 \mathrm{~h}$ before UV irradiation. This procedure is necessary for cell adhesion and to assure the irradiation of the majority of isolated cells. Cells were washed twice with prewarmed PBS and irradiated by a low-pressure germicidal lamp (UV light emitting mainly at $254 \mathrm{~nm}$ ). Photoreactivation (PRL) was performed for $2 \mathrm{~h}$ in PBS, except when otherwise indicated. Single cell layers were illuminated $10 \mathrm{~cm}$ over fluorescent lights (two daylight lamps, Philips $15 \mathrm{~W}$; emission, 400-700 nm). The cells were kept at $37^{\circ} \mathrm{C}$. After treatment, cells were maintained in a complete medium for 7-10 days and then fixed with $10 \%$ formaldehyde and stained with $1 \%$ violet crystal. Colonies with the minimal number of 15 cells were scored. Survival values were obtained as the ratio of the number of colonies from irradiated cells to non-irradiated cells.

\section{Flow cytometry Analysis}

Approximately $1.0 \times 10^{6}$ cells were plated in $100-\mathrm{mm}$ Petri dishes. After $24 \mathrm{~h}$, the cells were UV irradiated and maintained in dark or photoreactivating light conditions (PRL) in prewarmed PBS for $2 \mathrm{~h}$. Cells were trypsinized $48 \mathrm{~h}$ after the UV treatment and centrifuged at 1500 r.p.m. for $10 \mathrm{~min}$. Pelleted cells $\left(0.5 \times 10^{6}\right.$ to $1.0 \times 10^{6}$ cells $)$ were lysed with $500 \mu$ l of a hypotonic fluorochrome solution $(50 \mu \mathrm{g} / \mathrm{ml} \mathrm{PI}$ in $0.1 \%$ sodium citrate plus $0.1 \%$ Triton $\mathrm{X}-100$ ), and incubated at least 30 min on ice in the dark. ${ }^{31}$ Then, samples were transferred to microtubes, and PI fluorescence was measured by flow cytometry (FACScalibur, Becton Dickinson). Results were obtained as the percentage of subdiploid nuclei (CellQuest Software, Becton Dickinson), which represent the apoptotic cells.

\section{Measurement of RNA synthesis}

10000 to 15000 cells were plated on coverslips in a normal medium. After $24 \mathrm{~h}$ the medium was replaced by DMEM with 3\% FBS. After $24 \mathrm{~h}$ the cells were irradiated and maintained in dark or PRL conditions in prewarmed PBS for $1 \mathrm{~h}$ after different periods following UV, and maintained during $1 \mathrm{~h}$ in a medium with $3 \%$ FBS. Nascent RNA was then pulse-labeled in a medium containing ${ }^{3} \mathrm{H}$-uridine $(60 \mu \mathrm{Ci} / \mathrm{ml}$, Amersham Pharmacia Biotech, USA) and 3\% dialyzed FBS during $1 \mathrm{~h}$. The cells were washed with PBS, fixed in methanol : acetic acid $(3: 1)$, washed three times with 15\% TCA and once with ethanol. The cells were exposed to autoradiography as described. ${ }^{32}$ The silver grains corresponding to RNA transcription were counted to 50 nuclei in each sample. Grain counting was performed under systematically selected randomly optical fields from multiple noncontiguous sectors spanning the coverslips. The relative levels of transcription were obtained for each sample as the ratio of the mean grain count number in nuclei from UV irradiated cells to the number obtained in non-irradiated cells.

\section{Northern blot experiments}

Cells were plated in semiconfluence $\left(\cong 1.5 \times 10^{6}\right.$ cells/plate) and then harvested for RNA extraction. The total RNA was extracted with phenol/chloroform/isoamyl alcohol, precipitated with ethanol and then resuspended in $50 \mu \mathrm{l} \mathrm{H}_{2} \mathrm{O}$ containing diethyl pyrocarbonate (DEPC,
ICN). Samples containing $5 \mu \mathrm{g}$ of RNA were resolved by denaturing electrophoresis, and then transferred to a nylon membrane and hybridized with ${ }^{32} \mathrm{P}$-labeled DNA probe (photolyase and G6PDH genes) by standard methods. ${ }^{33}$ The hybridized bands were visualized by autoradiography.

\section{Measurement of UV endonuclease sensitive sites (ESS)}

Approximately $1.0 \times 10^{6}$ cells were plated in $60-\mathrm{mm}$ Petri dishes. After $24 \mathrm{~h}$, the cells were grown in complete medium containing ${ }^{3} \mathrm{H}$-methylthymidine $(0.25 \mu \mathrm{Ci} / \mathrm{ml}$, Amersham-Pharmacia Biotech, USA) for $24 \mathrm{~h}$. After this period, the cells were UV irradiated and maintained in dark or photoreactivating light conditions (PRL) in pre-warmed PBS for $1 \mathrm{~h}$. Nuclei were prepared immediately after this treatment with $0.5 \%$ Triton $X-100,0.1 \mathrm{M} \mathrm{NaCl}$ and $10 \mathrm{mM}$ EDTA, washed twice with PBS, and incubated in NET buffer $(100 \mathrm{mM} \mathrm{NaCl}, 10 \mathrm{mM}$ Tris- $\mathrm{HCl}$ and $10 \mathrm{mM}$ EDTA) (30), with and without T4-endonuclease V, for $30 \mathrm{~min}$ at $37^{\circ} \mathrm{C}$. Molecular weights of untreated and treated DNA were determined by alkaline sucrose gradient sedimentation, as described before. ${ }^{15}$ From these values, the number of T4-endonuclease sensitive-sites (ESS), which corresponds to the number of CPDs per $10^{7}$ daltons, was calculated.

\section{Acknowledgments}

We would like to thank Dr. Eliane N Miyaji (Inst. Butantan, São Paulo, Brazil) for scientific discussion and critical review of the manuscript, Dr. Junnichi Miyazaki (Osaka University, Japan) for providing the phr gene and FAPESP for financial support (São Paulo, Brazil). CFM Menck and GP Amarante-Mendes have research fellowships from CNPq (Brasília, Brasil), V Chiganças and $G$ Brumatti have graduation fellowships from FAPESP and LFZ Batista from CNPq.

\section{References}

1. Miyaji EN and Menck CFM (1996) Photoreversion of ultraviolet induced apoptosis in rat kangaroo cells. Apoptosis 1: 153-160

2. Chiganças V, Miyaji EN, Muotri AR, Jacsyn JF, Amarante-Mendes GP, Yasui A and Menck CFM (2000) Photorepair prevents ultraviolet-induced apoptosis in human cells expressing the marsupial photolyase gene. Cancer Res. 60: 24582463

3. Ma L, Hoeijmakers, JHJ and van Eb AJ (2000) Mammalian nucleotide excision repair. Biochim. Biophys. Acta 1242: 137-164

4. de Boer J and Hoeijmakers JHJ (2000) Nucleotide excision repair and human syndromes. Carcinogenesis 21: 453-460

5. Machado CR and Menck CFM (1997) Human DNA Repair diseases: From genomic instability to cancer. Braz. J. Genet. 20: 755-762

6. Ljungman M and Zhang F (1996) Blockage of RNA polymerase as a possible trigger for UV light- induced apoptosis. Oncogene 13: 823-831

7. Amarante-Mendes GP and Green DR (1999) The regulation of apoptotic cell death. Braz. J. Med. Biol. Res. 32: 45-61

8. Myiaji EN and Menck CFM (1995) Ultraviolet-induced cell death is independent of DNA replication in rat kangaroo cells. Photochem. Photobiol. 61: 454-458

9. Dunkern TRand Kaina B (2002) Cell proliferation and DNA breaks are involved in ultraviolet light-induced apoptosis in nucleotide excision repair-deficient Chinese hamster cells. Mol. Biol. Cell 13: 348-361.

10. Wang XW, Ermeulin W, Coursen JD, Gibson M, Lupold SE, Forrester K, Xu G, Elmore L, Yeh H, Hoeijmakers JHJ and Harris CC (1996) The XPB and XPD DNA helicases are components of the $\mathrm{p53}$-mediated apoptosis pathway. Genes Dev. 10: $1219-1232$ 
11. Donahue BA, Yin S, Taylor JS, Reines D and Hanawalt PC (1994) Transcript cleavage by RNA polymerase II by a cyclobutane pyrimidine dimer in the DNA template. Proc. Natl. Acad. Sci. USA 91: 8502-8506

12. Mckay BC, Ljungman M and Rainbow AJ (1998) Persistent DNA damage induced by ultraviolet light inhibits p $21^{\text {waf1 } 1}$ and bax expression: implications for DNA repair, UV sensitivity and the induction of apoptosis. Oncogene 17: $545-$ 555

13. Ljungman M (1999) Recovery of RNA synthesis from the DHFR gene following UV-irradiation precedes the removal of photolesions from the transcribed strand. Carcinogenesis 20: 395-399

14. Mckay BC, Chen F, Clarke ST, Wiggin HE, Harley LM and Ljungman M (2001) UV-light induced degradation of RNA polymerase II is dependent on the Cockayne's syndrome A and B proteins but not p53 or MLH1. Mutat. Res. 485: 93-105

15. Menck CFM and Meneghini R (1982) Recovery in the survival capacity of ultraviolet-irradiated $3 T 3$ mouse cells at $G_{0}$ cannot be solely dependent on the excision of pyrimidine dimers. Mutat. Res. $96: 273-280$

16. Amarante-Mendes GP, Finucane DM, Martin SJ, Cotter TG, Salvesen GS and Green DR (1998) Anti-apoptotic oncogenes prevent caspase-dependent and independent commitment for cell death. Cell Death Differ. 5: 298-396

17. Asashina H, Han ZB, Kawanishi M, Kato Jr T, Ayaki H, Todo T, Yagi T, Takebe, Ikenaga M and Kimura SH (1999) Expression of a mammalian DNA photolyase confers light-dependent repair activity and reduces mutations of UV-irradiated shuttle vectors in xeroderma pigmentosum cells. Mutat. Res. 435: 255-262

18. You YH, Lee DH, Yoon JH, Nakajima S, Yasui A and Pfeifer G (2001) Cyclobutane pyrimidine dimers are responsible for the vast majority of mutations induced by UVB irradiation in mammalian cells. J. Biol. Chem. 276: 4468844694

19. Rockx DA, Mason R, van Hoffen A, Barton MC, Citterio E, Bregman DB, van Zeeland AA, Vrieling $\mathrm{H}$ and Mullenders LH (2000) UV-induced inhibition of transcription involves repression of transcription initiation and phosphorylation of RNA polymerase II. Proc. Natl. Acad. Sci. USA 97: 10503-10508

20. Selby CP, Drapkin R, Reinberg D and Sancar A (1997) RNA polymerase stalled at a thymine dimer: footprint and effect on excision repair. Nucleic Acids Res 25: $787-793$

21. Tornaletti S, Reines D and Hanawalt PC (1999) Structural characterization of RNA polymerase II complexes arrested by a cyclobutane pyrimidine dimer in the transcribed strand of template DNA. J. Biol. Chem. 274: 24124-24130

22. Mellon I, Spivak G and Hanawalt PC (1987) Selective removal of transcriptionblocking DNA damage from the transcribed strand of the mammalian DHFR gene. Cell 51: 241-249
23. van Gool AJ, Citterio E, Rademakers $S$, van Os R, Vermeulen W, Constantinou A, Egly JM, Bootsma D and Hoeijmakers JHJ (1997) The Cockayne syndrome B protein, involved in transcription-coupled DNA repair, resides in an RNA polymerase Il-containing complex. EMBO J. 16: 5955-5965

24. Tantin D, Kansal A and Carey M (1997) Recruitment of the putative transcriptionrepair coupling factor CSB/ERCC6 to RNA polymerase Il elongation complexes. Mol. Cell Biol. 17: 6803-6814

25. le Page F, Kwoh EE, Avrutskya A, Gentil A, Leadon SA, Sarasin A and Cooper $P$ (2000) Transcription-coupled repair of 8-oxoguanine: requirement for XPG, TF $\mathrm{IIH}$, and CS-B and implications for Cockayne syndrome. Cell 101: 159-171

26. Volker M, Moné MJ, Karmakar $\mathrm{P}$, van Hoffen A, Schul W, Vermeulen W, Hoeijmakers JHJ, van Driel R, van Zeeland AA and Mullenders LHF (2001) Sequential assembly of the nucleotide excision repair factors in vivo. Mol. Cell 8 : 213-224

27. Svejstrup JQ, Vichi P and Egly JM (1996) The multiple roles of transcription/ repair factor TFIIH. Trends Biochem. Sci. 21: 346-350

28. Tantin D (1998) RNA polymerase II elongation complexes containing the Cockayne syndrome group B protein interact with a molecular complex containing the transcription factor $\mathrm{IHH}$ components Xeroderma pigmentosum group B and p62. J. Biol. Chem. 273: 27794-27799

29. van Hoffen $A$, Kalle WH, de Jong-Versteeg A, Lehmann AR, van Zeeland $A A$ and Mullenders LH (1999) Cells from XP-D and XP-D CS patients exhibit equally inefficient repair of UV-induced damage in transcribed genes but differen capacity to recover UV-inhibited transcription. Nucleic Acids Res. 27: 2898 2904

30. Felgner PL, Gadek TR, Holm M, Roman R, Chan HW, Wenz M, Northrop JP, Ringold GM and Danielsen M (1987) Lipofectin: a highly efficient, lipid-mediated DNA-transfection procedure. Proc. Natl. Acad. Sci. USA 84: 7413-7417

31. Amarante-Mendes GP, Bossy-Wetzel E, Brunner T and Green DR (1998) Apoptosis Assays. In Cell: A Laboratory Manual, Spector DL, Goldman R and Leinwand L, eds (CSHL: Cold Spring Harbor Laboratory Manual on Cell Biology) pp. $15.1-15.19$

32. Yamaizumi M and Sugano T (1994) U.v.-induced accumulation of $p 53$ is evoked through DNA damage of actively transcribed genes independent of the cell cycle. Oncogene 9: 2775-2784

33. SambrookJ and Russel DW (eds) (2001) Transfer and fixation of denatured RNA to membranes. In Molecular Cloning: A Laboratory Manual, (New York: Cold Spring Harbor Laboratory Press) pp. 7.35-7.45 